\title{
LIMIT CYCLES FOR DISCONTINUOUS PLANAR PIECEWISE LINEAR DIFFERENTIAL SYSTEMS SEPARATED BY AN ALGEBRAIC CURVE
}

\author{
JAUME LLIBRE ${ }^{1}$ AND XIANG ZHANG ${ }^{2}$
}

\begin{abstract}
We study how can change the maximum number of limit cycles of the discontinuous piecewise linear differential systems with only two pieces in function of the degree of the discontinuity algebraic curve between the two linear differential systems. These discontinuous differential systems appear frequently in applied sciences.
\end{abstract}

\section{INTRODUCTION AND STATEMENT OF THE MAIN RESUlt}

The dynamics of the piecewise linear differential systems started to be studied around 1930, mainly in the book of Andronov et al [1]. Many researchers from different fields considered this kind of differential systems, because they are widely used to model phenomena appearing in mechanics, electronics, economy, neuroscience, ..., see for more details and applications the books of Bernardo et al [3] and of Simpson [33], the surveys of Makarenkov and Lamb [29], and Teixeira [36], the articles [19, 20,21, 31,35], and the references which appear in all these works.

A periodic orbit of a differential system which is isolated in the set of all periodic orbits of the system is a limit cycle. One of the main problems in the dynamics of the differential systems in the plane is to control the existence and the number of their limit cycles. This problem restricted to polynomial differential systems is the famous 16th Hilbert's problem, see more details in [14, 18, 22].

This last years many authors have studied the limit cycles of discontinuous piecewise linear differential systems in $\mathbb{R}^{2}$. Of course, the simplest piecewise linear differential systems in $\mathbb{R}^{2}$ are the ones having only two pieces separated by a curve, and when this curve is a straight line. Thus the limit cycles of these last class of discontinuous piecewise linear differential systems has been intensively studied, see $[2,4,6,7,9,10,11,12,13,15,16,17,23,24,25,26,27,28,32]$ and the references quoted in these papers. Up to know the results of all these papers only provide examples that the discontinuous piecewise linear differential systems in $\mathbb{R}^{2}$ separated by a straight line can have 3 crossing limit cycles (they are defined in what follows), but it is an open question to know if 3 is the maximum number of crossing limit cycles that such discontinuous differential systems can have.

2010 Mathematics Subject Classification. Primary 34A30, 34C05, 34C25, 34C07, $37 \mathrm{G} 15$.

Key words and phrases. non-smooth differential system, limit cycle, discontinuous piecewise linear differential system. 
In the papers $[5,30]$ the authors considered discontinuous piecewise linear differential systems with only two pieces, but now the curve of discontinuity between the two pieces is not a straight line, and they shown that the number of limit cycles can increase arbitrarily with the number of oscillations of that curve. We note that the discontinuity curves considered in these two papers is not algebraic.

The main objective of this paper is to study how can change the maximum number of limit cycles of the discontinuous piecewise linear differential systems with only two pieces in function of the degree of the discontinuity curve when this curve is algebraic. Due to the big amount of computations necessary for studying this maximum number of limit cycles we restrict our analysis as follows: first to the class of discontinuous piecewise linear differential systems separated by the algebraic curve $y=x^{n}$ where $n>1$ is an arbitrary positive integer; second to the class of discontinuous piecewise linear differential systems formed by two linear differential systems having only centers; and third we only consider crossing limit cycles, they are defined in what follows.

We consider discontinuous piecewise differential equations in $\mathbb{R}^{2}$ of the form

$$
\left(\begin{array}{c}
\dot{x} \\
\dot{y}
\end{array}\right)= \begin{cases}X^{-}(x, y) & \text { if }(x, y) \in L=\left\{(x, y): y \leq x^{n}\right\} \\
X^{+}(x, y) & \text { if }(x, y) \in U=\left\{(x, y): y \geq x^{n}\right\}\end{cases}
$$

where the vector fields $X^{-}$and $X^{+}$are linear. The dynamics over the curve of discontinuity $y=x^{n}$ is defined following the Filippov's convention, see [8]. Thus, on the curve of discontinuity $y=x^{n}$ we distinguish generically two different kind of points: the crossing points $\left\{p=\left(x, x^{n}\right): X^{-}(p) X^{+}(p)>0\right\}$, and the sliding points $\left\{p=\left(x, x^{n}\right): X^{-}(p) X^{+}(p)<0\right\}$.

We assume that each one of the linear differential systems of the discontinuous piecewise linear differential systems (1) has a center. We want to find upper bound for the maximum number of crossing limit cycles that such discontinuous piecewise linear differential systems can exhibit. A crossing limit cycle for such discontinuous differential systems is formed by two pieces of orbits, one piece of orbit of the linear differential system contained in $y<x^{n}$ and the other piece of orbit of the linear differential system contained in $y>x^{n}$, furthermore both pieces connect in two crossing points over the curve $y=x^{n}$. We must mention that when $n=1$, i.e. when the discontinuous curve is a straight line, and both linear differential systems have a center the discontinuous differential system (1) has no crossing limit cycles, see for details [28]. This proves the result of the next Theorem 2 for the particular case $n=1$.

An equilibrium $p$ of a linear differential system of a discontinuous piecewise linear differential systems in $\mathbb{R}^{2}$ separated by the algebraic curve $y=x^{n}$ is called real when $p$ belongs to the closure of the component of $\mathbb{R}^{2} \backslash\left\{(x, y): y=x^{n}\right\}$ where is defined the mentioned linear differential system, otherwise $p$ is called virtual.

The following normal form for the discontinuous piecewise linear differential systems in $\mathbb{R}^{2}$ separated by the algebraic curve $y=x^{n}$ when both linear differential systems have a center will help us to prove our manin result, the theorem which follows the next proposition. 
Proposition 1. After a linear change of variables and a rescaling of the independent variable any discontinuous piecewise linear differential systems in $\mathbb{R}^{2}$ separated by the algebraic curve $f(x, y)=0$ when both linear differential systems have a center can be written as

$\begin{array}{lll}\dot{x}=-b x-\frac{4 b^{2}+\omega^{2}}{4 a} y+d, & \dot{y}=a x+b y+c, & \text { in } L=\left\{(x, y): y \leq x^{n}\right\}, \\ \dot{x}=-y-\alpha, & \dot{y}=x-\beta, & \text { in } U=\left\{(x, y): y \geq x^{n}\right\},\end{array}$

with $a>0$ and $\omega>0$.

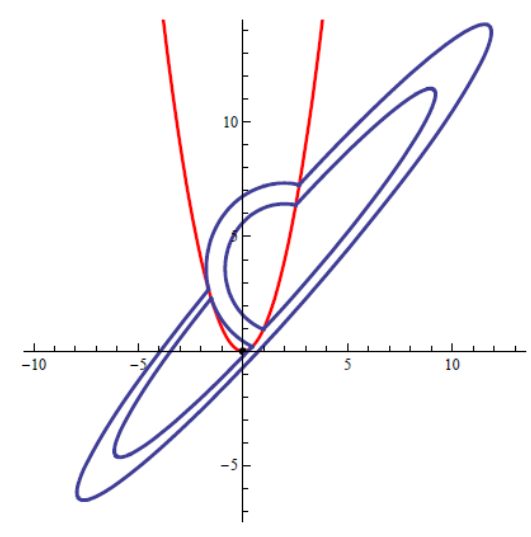

FIGURE 1. The three limit cycles of a discontinuous piecewise linear differential system (2) for $n=2$.

Theorem 2. Let $n$ be a positive integer.

(a) An upper bound for the maximum number of crossing limit cycles that a discontinuous piecewise linear differential system in $\mathbb{R}^{2}$ separated by the algebraic curve $y=x^{n}$ can have when both linear differential systems have a center, real or virtual, is $d_{n}$ equal to

$$
\frac{2 n^{2}-n-1}{2} \text { if } n \text { is odd, and } \frac{2 n^{2}-n}{2} \text { if } n \text { is even. }
$$

(b) For $n=2$ there are discontinuous piecewise linear differential systems which reach the upper bound of 3 limit cycles of statement $(a)$, see the 3 limit cycles in Figure 1.

(c) For $n=2,3$ we provide the explicit polynomial of degree $2 d_{n}$ whose real roots $x$ provide the points $\left(x, x^{n}\right)$ where the crossing limit cycles of the discontinuous piecewise linear differential system (2) intersect the curve $y=x^{n}$.

Theorem 2 is proved in section 3 .

We remark that in statement (a) of Theorem 2 we provide upper bound for the maximum number of crossing limit cycles that the discontinuous piecewise linear differential systems studied in that theorem can have. The major part of the 
results mentioned previously on the limit cycles of discontinuous piecewise linear differential systems studied until now only provide lower bounds for that maximum number. The unique other three papers that we know providing upper bounds are when the curve of discontinuity is a straight line, and either one of the linear differential systems has an equilibrium on the the discontinuity straight line [7, 24], or one of the linear differential systems has a center [28].

It remains the following open question.

Open question. Are the upper bounds given in the statement of Theorem 2 reached for values of $n>2$ ?

\section{NORMAL FORM}

Now we shall prove the normal form for the discontinuous piecewise linear differential systems that we study.

Proof of Proposition 1. Doing the linear change of variables which writes the matrix of the linear differential system in $U$, having a real or virtual center, in its real normal form, and rescaling the independent variable of the differential system (if necessary) we can write such a linear differential system as $\dot{x}=-y-\alpha, \dot{y}=x-\beta$.

Now the linear differential system in the region $L$ is

$$
\dot{x}=A x+B y+d, \quad \dot{y}=a x+b y+c,
$$

assuming that it has a center. Since the eigenvalues of this system are

$$
\frac{A+b \pm \sqrt{4 a B+(A-b)^{2}}}{2}
$$

in order to have a center we must have that $A+b=0$ and $4 a B+(A-b)^{2}=-\omega^{2}$ for some $\omega>0$ and $a B<0$, or equivalently $A=-b, B=-\left(4 b^{2}+\omega^{2}\right) /(4 a)$ and $a>0$. This completes the proof of the proposition.

It is easy to check that the differential linear system in the region $L$ has the first integral

$$
H_{L}=4(a x+b y)^{2}+8 a(c x-d y)+\omega^{2} y^{2},
$$

while the first integral of the differential linear system in the region $U$ is

$$
H_{U}=(y+\alpha)^{2}+(x-\beta)^{2} .
$$

\section{Proofs of Theorem 2}

In order that the piecewise linear differential system (2) has a crossing limit cycle $\Gamma$, it must intersect the discontinuous curve $y=x^{n}$ in two points. Let $\left(x_{L}, x_{L}^{n}\right)$ and $\left(x_{R}, x_{R}^{n}\right)$ be two intersecting points. Then, taking into account that $H_{L}$ and $H_{R}$ are first integrals these two points satisfy the equations

$$
\begin{aligned}
& e_{L}:=H_{L}\left(x_{L}, x_{L}^{n}\right)-H_{L}\left(x_{R}, x_{R}^{n}\right)=0, \\
& e_{U}:=H_{U}\left(x_{L}, x_{L}^{n}\right)-H_{U}\left(x_{R}, x_{R}^{n}\right)=0 .
\end{aligned}
$$


Note that $e_{L}$ and $e_{U}$ are two polynomials of degree $2 n$ in the variables $x_{L}$ and $x_{R}$. Clearly $x_{L}-x_{R}$ is a factor of both $e_{L}$ and $e_{R}$. Some direct calculations show that $E_{L}=e_{L} /\left(x_{L}-x_{R}\right)$ and $E_{R}=e_{R} /\left(x_{L}-x_{R}\right)$ are given by

$$
\begin{aligned}
E_{L}= & \left(4 b^{2}+\omega^{2}\right)\left(x_{R}^{2 n-1}+x_{L} x_{R}^{2 n-2}+\ldots+x_{L}^{n-3} x_{R}^{n+2}+x_{L}^{n-2} x_{R}^{n+1}\right) \\
& +\left(\left(4 b^{2}+\omega^{2}\right) x_{L}^{n-1}+8 a b\right) x_{R}^{n}+\left(\left(4 b^{2}+\omega^{2}\right) x_{L}^{n}+8 a b x_{L}-8 a d\right) x_{R}^{n-1} \\
& +\left(\left(4 b^{2}+\omega^{2}\right) x_{L}^{n+1}+8 a b x_{L}^{2}-8 a d x_{L}\right) x_{R}^{n-2} \\
& +\ldots+\left(\left(4 b^{2}+\omega^{2}\right) x_{L}^{2 n-3}+8 a b x_{L}^{n-2}-8 a d x_{L}^{n-3}\right) x_{R}^{2} \\
& +\left(\left(4 b^{2}+\omega^{2}\right) x_{L}^{2 n-2}+8 a b x_{L}^{n-1}-8 a d x_{L}^{n-2}+4 a^{2}\right) x_{R} \\
& +\left(4 b^{2}+\omega^{2}\right) x_{L}^{2 n-1}+8 a b x_{L}^{n}-8 a d x_{L}^{n-1}+4 a^{2} x_{L}+8 a c,
\end{aligned}
$$

and

$$
\begin{aligned}
E_{U}= & x_{R}^{2 n-1}+x_{L} x_{R}^{2 n-2}+\ldots+x_{L}^{n-3} x_{R}^{n+2}+x_{L}^{n-2} x_{R}^{n+1}+x_{L}^{n-1} x_{R}^{n} \\
& +\left(x_{L}^{n}+2 \alpha\right)\left(x_{R}^{n-1}+x_{L} x_{R}^{n-2}+\ldots+x_{L}^{n-3} x_{R}^{2}\right) \\
& +\left(\left(x_{L}^{n}+2 \alpha\right) x_{L}^{n-2}+1\right) x_{R}+\left(x_{L}^{n}+2 \alpha\right) x_{L}^{n-1}+x_{L}-2 \beta .
\end{aligned}
$$

Note that $E_{L}$ and $E_{U}$ are polynomials in the variables $x_{L}$ and $x_{R}$ of degree $2 n-1$.

To study the existence and the number of limit cycles of system (2), it is necessary to compute the common zeros $\left(x_{L}, x_{R}\right)$ of $E_{L}$ and $E_{R}$. For doing so we calculate the resultants Resultant $\left[E_{L}, E_{R}, x_{R}\right]$ and Resultant $\left[E_{L}, E_{R}, x_{L}\right]$ of $E_{L}$ and $E_{R}$ with respect to $x_{R}$ and $x_{L}$, respectively. By the symmetry of $E_{L}$ and $E_{R}$ with respect to $x_{L}$ and $x_{R}$, we know that Resultant $\left[E_{L}, E_{R}, x_{R}\right]$ and Resultant $\left[E_{L}, E_{R}, x_{L}\right]$ have the same expression. So we only need to compute Resultant $\left[E_{L}, E_{R}, x_{R}\right]$.

In what follows we denoted by $R_{L}$ (respectively $R_{R}$ ) for simplifying the notation the Resultant $\left[E_{L}, E_{R}, x_{R}\right]$ (respectively Resultant $\left[E_{L}, E_{R}, x_{L}\right]$ ) because this resultant is a polynomial in the variable $x_{L}$ (respectively $x_{R}$ ).

The next result provides the degree of the resultant $R_{L}$, which is the most technical lemma in the proof of Theorem 2. We remark that in general the degree of the resultant of two arbitrary polynomials of degree $2 n-1$ is $(2 n-1)^{2}$, but since the polynomials $E_{L}$ and $E_{R}$ are special the degree in this case is lower, and to compute this degree is the more difficult part of this paper.

Lemma 3. The maximum degree of the resultant $R_{L}$ is

$$
2 n^{2}-n-1 \text { if } n \text { is odd, and } 2 n^{2}-n \text { if } n \text { is even. }
$$

Proof. Let $S_{0}=4 b^{2}+\omega^{2}, S_{1}=-8 a d, S_{2}=4 a^{2}, S_{3}=8 a c$ and $A=8 a b$. And for simplifying notations we replace $x_{L}$ by $x$. Then by the definition of the resultant of two polynomials $R_{L}$ is the determinant of a $(4 n-2) \times(4 n-2)$ matrix, more precisely

$$
R_{L}=\left|\begin{array}{cccccccccccc}
C_{1} & 0_{1} & \cdots & 0_{n-1} & \cdots & 0_{2 n-2} & C_{2} & 0_{1} & \cdots & 0_{n-1} & \cdots & 0_{2 n-2} \\
& C_{1} & & & & & & C_{2} & & & & \\
& & \ddots & & & & & & \ddots & & & \\
& & & C_{1} & & & & & & C_{2} & & \\
& & & & \ddots & & & & & & \ddots & \\
& & & & & C_{1} & & & & & C_{2}
\end{array}\right|,
$$


where $0_{k}$ is a $k$-dimensional column vector for $k \in\{1, \ldots, n-2\}$, the entries which are empty are zeros, and $C_{1}$ and $C_{2}$ are $2 n$-dimensional column vectors given respectively as

$$
C_{1}=\left[\begin{array}{c}
S_{0} \\
S_{0} x \\
S_{0} x^{2} \\
\vdots \\
S_{0} x^{n-3} \\
S_{0} x^{n-2} \\
S_{0} x^{n-1}+A \\
S_{0} x^{n}+A x+S_{1} \\
S_{0} x^{n+1}+A x^{2}+S_{1} x \\
\vdots \\
S_{0} x^{2 n-3}+A x^{n-2}+S_{1} x^{n-3} \\
S_{0} x^{2 n-2}+A x^{n-1}+S_{1} x^{n-2}+S_{2} \\
S_{0} x^{2 n-1}+A x^{n}+S_{1} x^{n-1}+S_{2} x+S_{3}
\end{array}\right], C_{2}=\left[\begin{array}{c}
1 \\
x \\
x^{2} \\
\vdots \\
x^{n-3} \\
x^{n-2} \\
x^{n-1} \\
x^{n}+2 \alpha \\
x^{n+1}+2 \alpha x \\
\vdots \\
x^{2 n-3}+2 \alpha x^{n-3} \\
x^{2 n-2}+2 \alpha x^{n-2}+1 \\
x^{2 n-1}+2 \alpha x^{n-1}+x-2 \beta
\end{array}\right] .
$$

For computing the determinant $R_{L}$, we first apply the next algorithm to $R_{L}$ :

- We add the $(2 n-1+j)$-th column multiplied by $-\left(4 b^{2}+\omega^{2}\right)$ to the $j$-th column for $j=1, \ldots, 2 n-1$.

Then

- We expand the resulting determinant of a $(4 n-2) \times(4 n-2)$ matrix through its first row.

- We expand the new resulting determinant of a $(4 n-3) \times(4 n-3)$ matrix through its first row.

- We expand this second new resulting determinant of a $(4 n-4) \times(4 n-4)$ matrix again through its first row.

- In total we repeat this process $n-1$ times, and at the end we have that $R_{L}$ is the determinant of a $(3 n-1) \times(3 n-1)$ matrix.

After all these computations we achieve that $R_{L}=(-1)^{n-1} R_{L_{1}}$ with

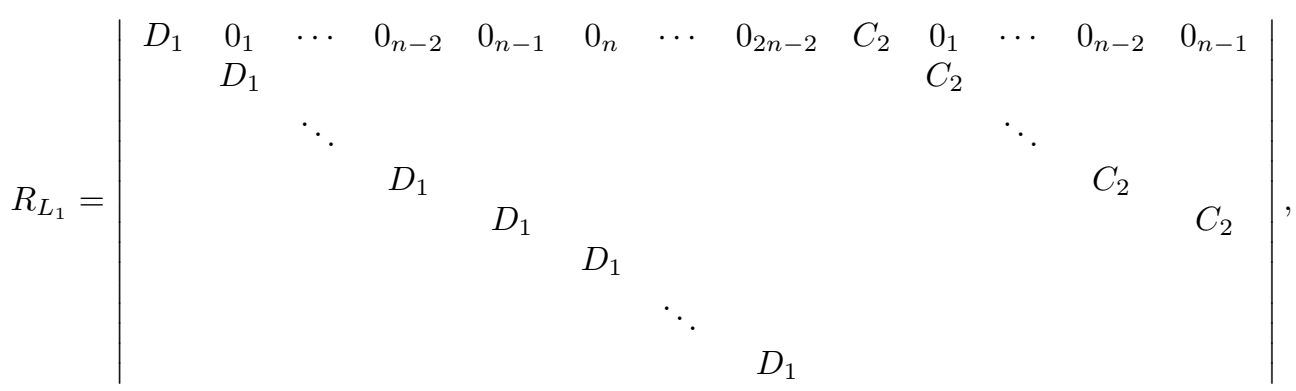


where the entries without letters are zeros, and $D_{1}$ is the $n+1$-dimensional column vector given by

$$
D_{1}=\left[\begin{array}{c}
A \\
A x+B \\
A x^{2}+B x \\
\vdots \\
A x^{n-2}+B x^{n-3} \\
A x^{n-1}+B x^{n-2}+C \\
A x^{n}+B x^{n-1}+C x+D
\end{array}\right]
$$

where $B=-8 a d-2 \alpha\left(4 b^{2}+\omega^{2}\right), C=4 a^{2}-\left(4 b^{2}+\omega^{2}\right)$ and $D=8 a c+2 \beta\left(4 b^{2}+\omega^{2}\right)$.

We separate the rest of the proof in three cases, first $n>3$, after $n=2$ and finally $n=3$.

We do the next computations with $R_{L_{1}}$ when $n>3$ :

- We add the first row multiplied by $-x^{\ell}$ to the $(\ell+1)$-th row for $\ell=1, \ldots, n$.

- In the new resulting determinant we add the second row multiplied by $-x^{\ell}$ to the $(\ell+2)$-th row for $\ell=1, \ldots, n-1$.

- In this second new resulting determinant we add the third row multiplied by $-x^{\ell}$ to the $(\ell+3)$-th row for $\ell=1, \ldots, n-2$.

- In this third new resulting determinant we add the fourth row multiplied by $-x^{\ell}$ to the $(\ell+3)$-th row for $\ell=1, \ldots, n-3$.

- In total we repeat this process $n$ times.

Now $R_{L_{1}}$ is the determinant of a $(3 n-1) \times(3 n-1)$ matrix of the form

$$
R_{L_{1}}=\left|\begin{array}{ll}
M_{1} & M_{2} \\
M_{3} & M_{4}
\end{array}\right|
$$

where $M_{1}, M_{2}, M_{3}$ and $M_{4}$ are respectively the $(n+1) \times(n+1),(n+1) \times(2 n-2)$, $(2 n-2) \times(n+1)$ and $(2 n-2) \times(2 n-2)$ matrices given by

$$
\begin{aligned}
& M_{1}=\left[\begin{array}{ccccccc}
A & & & & & & \\
B & A & & & & & \\
& B & \ddots & & & & \\
& & \ddots & \ddots & & & \\
& & & \ddots & \ddots & & \\
& & & & \ddots & A & \\
C & & & & & B & A
\end{array}\right] \text {, } \\
& M_{2}=\left[\begin{array}{cccccc}
0_{n-2}^{T} & 1 & 0 & & & \\
& & 1 & & & \\
\vdots & & & \ddots & & \\
& & & & 1 & 0 \\
0 & & & & & 1 \\
0 & 2 \alpha & & & & 0
\end{array}\right] \text {, }
\end{aligned}
$$




$$
M_{3}=\left[\begin{array}{cccccccc}
0_{2 n-2} & f_{n}(x) & g_{n-1}(x) & h_{n-2}(x) & h_{n-3}(x) & \cdots & \cdots & h_{1}(x) \\
& 0_{2 n-3} & f_{n}(x) & g_{n-1}(x) & h_{n-2}(x) & \ddots & \ddots & \vdots \\
& & 0_{2 n-4} & \ddots & \ddots & \ddots & \ddots & \vdots \\
& & & & \ddots & \ddots & \ddots & h_{n-3}(x) \\
& & & & & \ddots & \ddots & h_{n-2}(x) \\
& & & & & & \ddots & g_{n-1}(x) \\
& & & & & & & f_{n}(x) \\
& & & & & & & 0_{n-2}
\end{array}\right],
$$

where

$$
\begin{aligned}
f_{n}(x) & =A x^{n}+B x^{n-1}+C x+D, \\
g_{n-1}(x) & =A x^{n-1}+B x^{n-2}+C, \\
h_{j}(x) & =A x^{j}+B x^{j-1} \text { for } j=n-2, \ldots, 1,
\end{aligned}
$$

and $M_{4}$ is equal to

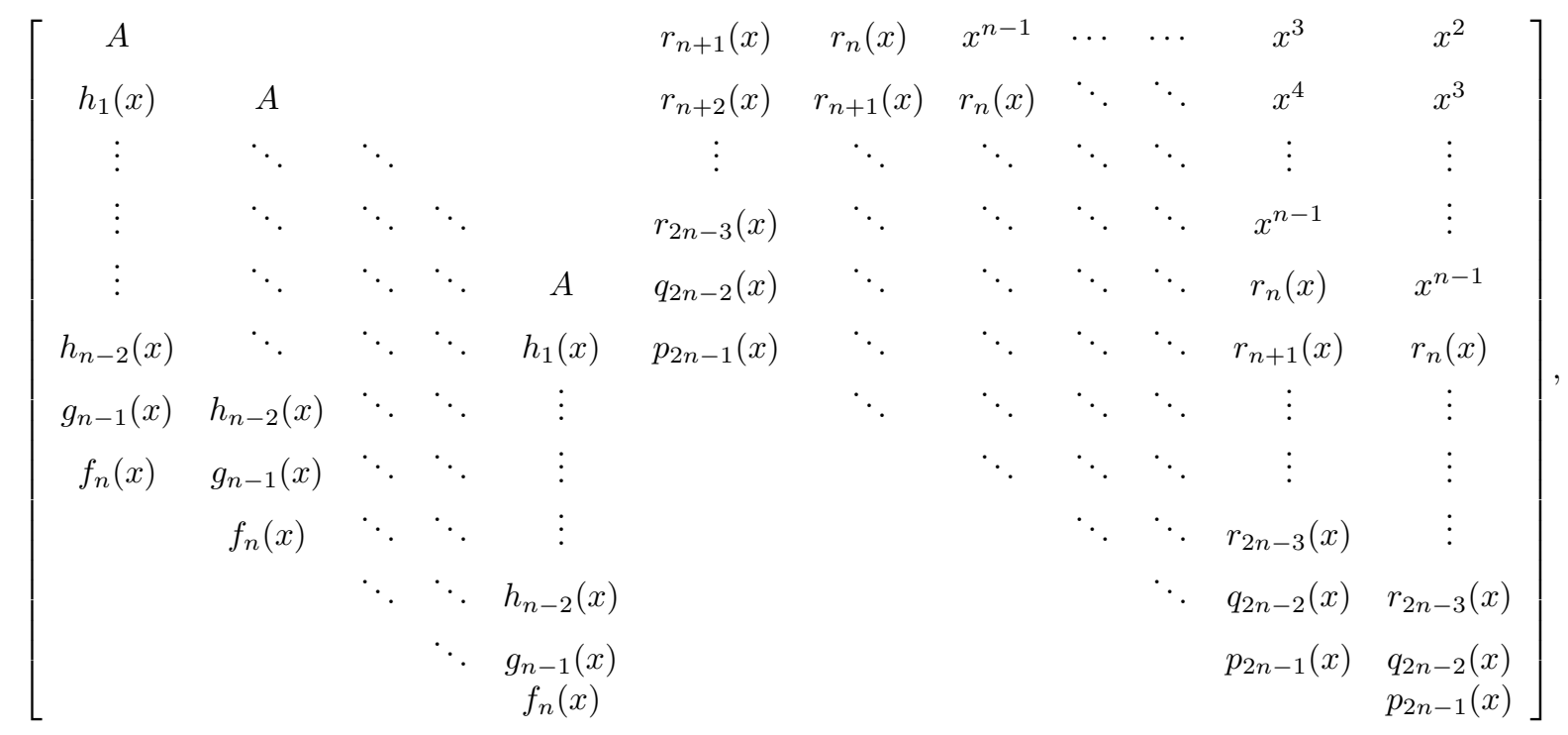

where

$$
\begin{aligned}
p_{2 n-1}(x) & =x^{2 n-1}+\left(2 \alpha x^{n-2}+1\right) x-2 \beta \\
q_{2 n-2}(x) & =x^{2 n-2}+2 \alpha x^{n-2}+1 \\
r_{j}(x) & =x^{j}+2 \alpha x^{j-n} \text { for } j=2 n-3, \ldots, n \text { with } j \geq 3 .
\end{aligned}
$$

Since $\operatorname{det} M_{1} \neq 0$ because $a b \neq 0$, doing some linear algebra we get that $\operatorname{det} R_{L_{1}}=\operatorname{det} M_{1} \operatorname{det}\left(M_{4}-M_{3} M_{1}^{-1} M_{2}\right)=(\operatorname{det} A)^{n+1} \operatorname{det}\left(M_{4}-M_{3} M_{1}^{-1} M_{2}\right)$. 
Direct calculations show that

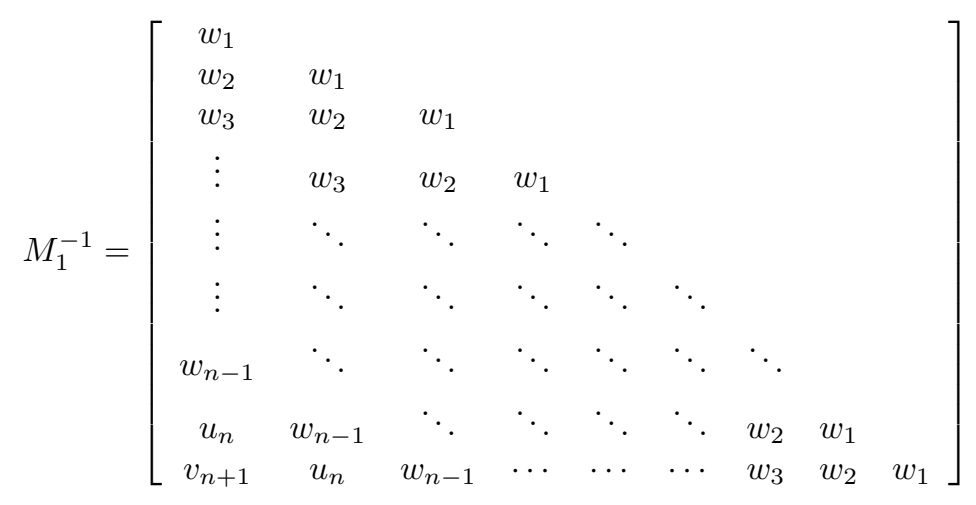

where

$$
\begin{aligned}
w_{k} & =(-1)^{k-1} A^{-k} B^{k-1} \quad \text { for } k=1,2, \ldots, n-1, \\
u_{n} & =(-1)^{n-1} A^{-n} B^{n-1}-A^{-2} C, \\
v_{n+1} & =(-1)^{n} A^{-(n+1)} B^{n}+2 A^{-3} B C-A^{-2} D .
\end{aligned}
$$

Furthermore,

$$
M_{1}^{-1} M_{2}=\left[\begin{array}{ccccccccc}
0_{n-2}^{T} & w_{1} & & & & & & & \\
0_{n-2}^{T} & w_{2} & w_{1} & & & & & & \\
0_{n-2}^{T} & w_{3} & w_{2} & w_{1} & & & & \\
\vdots & \vdots & w_{3} & w_{2} & w_{1} & & & & \\
\vdots & \vdots & \ddots & \ddots & \ddots & \ddots & & & \\
\vdots & \vdots & \ddots & \ddots & \ddots & \ddots & \ddots & & \\
0_{n-2}^{T} & w_{n-1} & \ddots & \ddots & \ddots & \ddots & \ddots & \ddots & \\
0_{n-2}^{T} & u_{n} & w_{n-1} & \ddots & \ddots & \ddots & \ddots & w_{2} & w_{1} \\
0_{n-2}^{T} & v_{n+1}+2 \alpha w_{1} & u_{n} & w_{n-1} & \cdots & \cdots & \cdots & w_{3} & w_{2}
\end{array}\right],
$$

and

$$
M_{3} M_{1}^{-1} M_{2}=\left[\begin{array}{cc}
0_{n \times(n-2)} & M_{n \times n} \\
0_{(n-2) \times(n-2)} & 0_{(n-2) \times n}
\end{array}\right], \quad M_{n \times n}=\left(F_{k \ell}\right)_{k, \ell=1, \ldots, n},
$$

with $0_{i \times j}$ the zero $i \times j$ matrix, and

$$
F_{k \ell}=\sum_{s=1}^{n+1-k} h_{k-1+s} w_{n+3-\ell-s}
$$

where for simplifying the notation we have used $w_{j}=0$ for $j \leq 0, h_{n}=f_{n}$, $h_{n-1}=g_{n-1}, w_{n}=u_{n}$ and $w_{n+1}=v_{n+1}+2 \alpha w_{1}$.

Since $\operatorname{det} A=8 a b \neq 0$, the computation of $R_{L_{1}}$ has been reduced to calculate the determinant of the matrix $M_{4}-M_{3} M_{1}^{-1} M_{2}$. Note that $M_{4}$ and $M_{3} M_{1}^{-1} M_{2}$ are $(2 n-2) \times(2 n-2)$ matrices. Now we work with these two matrices according to the following algorithm: 
- we add the $i$-th row multiplied by $-x$ to the $(i+1)$-th row for $i=n-3, n-$ $2, \ldots, 1$, successively.

At the end of this process the matrix $M_{4}$ has been reduced to the matrix $M_{4}^{*}$ given by

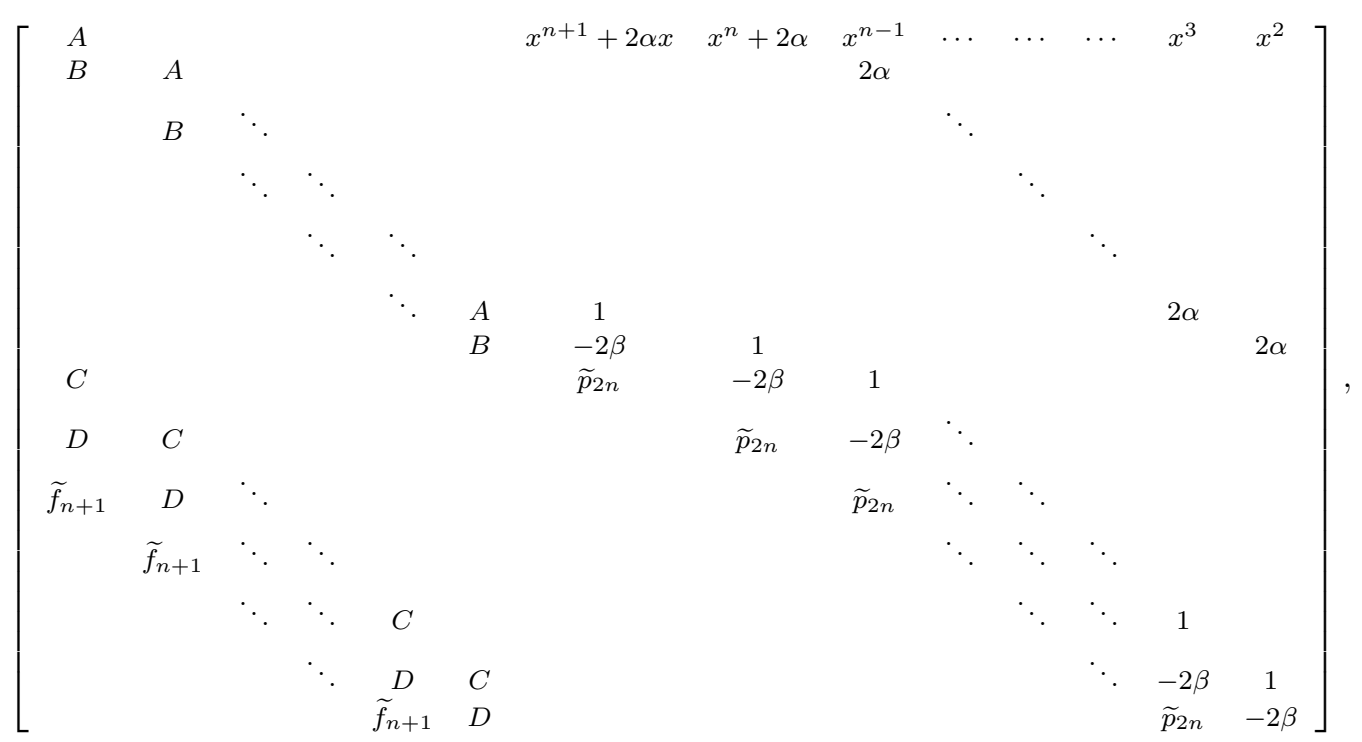

where $\widetilde{f}_{n+1}=-x f_{n}(x)$ and $\widetilde{p}_{2 n}=-x p_{2 n-1}(x)$ are polynomials of degrees $n+1$ and $2 n$ respectively. Applying the same algorithm to the matrix $M_{3} M_{1}^{-1} M_{2}$, this has been reduced to the matrix $M_{3}^{*}$ given by

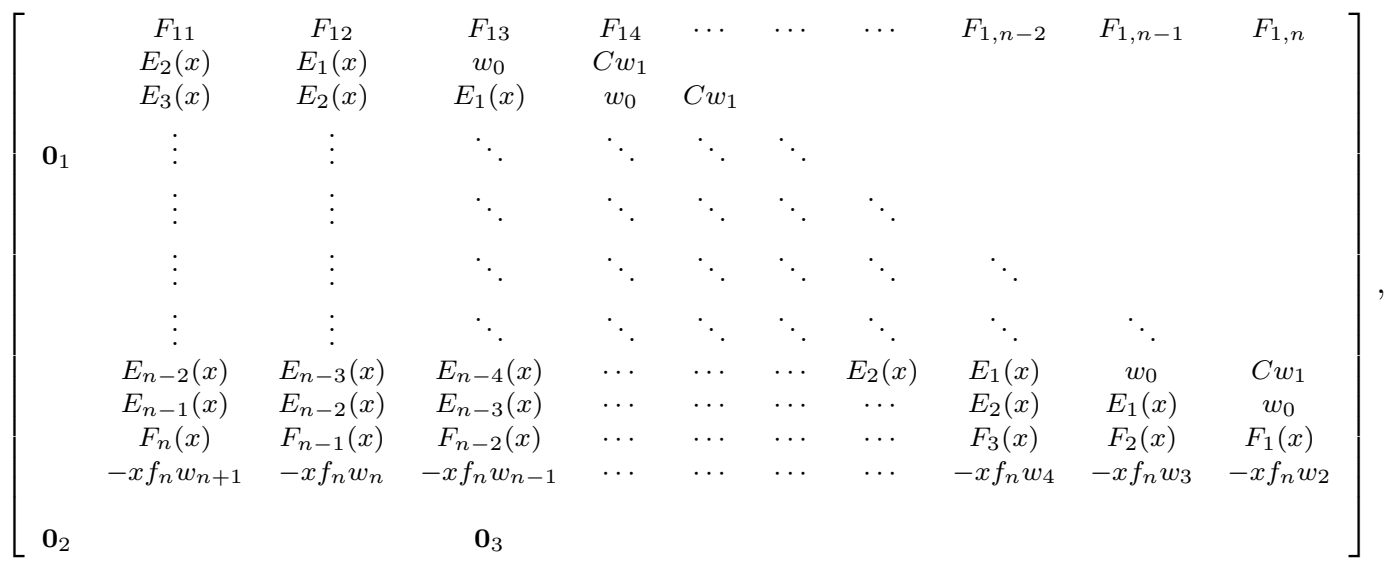


where $\mathbf{0}_{1}=0_{(n+1) \times(n-2)}, \mathbf{0}_{2}=0_{(n-3) \times(n-2)}, \mathbf{0}_{3}=0_{(n-3) \times n}, w_{0}=D w_{1}+C w_{2}$, and

$$
\begin{aligned}
E_{i}(x)= & -x f_{n}(x) w_{i}+D w_{i+1}+C w_{i+2} \quad \text { for } i=1, \ldots, n-1, \\
F_{i}(x)= & -x f_{n}(x) w_{i}+D w_{i+1}, \quad \text { for } i=1, \ldots, n, \\
F_{11}= & -A^{-1} B x^{n}+\left(A^{-2} B C-A^{-1} D+2 \alpha\right) x, \\
& \quad+(-1)^{n} A^{-n-1} B^{n+1}+2 A^{-3} B^{2} C-2 A^{-2} B D+2 \alpha A^{-1} D, \\
F_{12}= & x^{n}+(-1)^{n-1} A^{-n} B^{n}-A^{-2} B C+A^{-1} D, \\
F_{13}= & x^{n-1}+(-1)^{n-2} A^{-(n-1)} B^{n-1}+A^{-1} C, \\
F_{1 \ell}= & x^{n+2-\ell}+(-1)^{n+1-\ell} A^{-(n+2-\ell)} B^{n+2-\ell}, \quad \text { for } \ell=4, \ldots, n .
\end{aligned}
$$

Now $\operatorname{det}\left(M_{4}-M_{3} M_{1}^{-1} M_{2}\right)=\operatorname{det}\left(M_{4}^{*}-M_{3}^{*}\right)$, and this last determinant is the determinant of the matrix

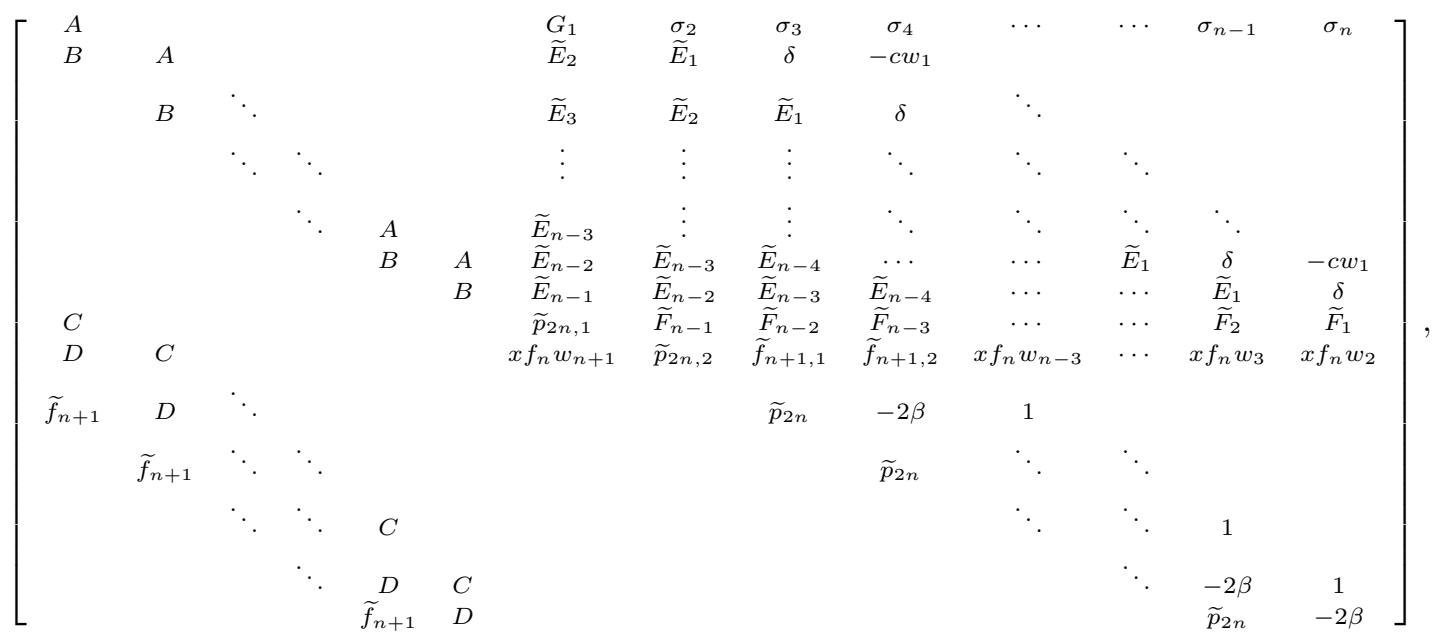

where the entries without anything are all zeros, and

$$
\begin{aligned}
& G_{1}=G_{1}(x)=x^{n+1}+A^{-1} B x^{n}+\left(A^{-2} B C-A^{-1} D\right) x \\
& -(-1)^{n} A^{-n-1} B^{n+1}-2 A^{-3} B^{2} C+2 A^{-2} B D-2 \alpha A^{-1} D, \\
& \sigma_{2}=2 \alpha+(-1)^{n} A^{-n} B^{n}+A^{-2} B C-A^{-1} D, \\
& \sigma_{3}=(-1)^{n-1} A^{-(n-1)} B^{n-1}-A^{-1} C \text {, } \\
& \sigma_{\ell}=(-1)^{n+2-\ell} A^{-(n+2-\ell)} B^{n+2-\ell}, \quad \ell=4, \ldots, n, \\
& \delta=2 \alpha-D w_{1}-C w_{2} \text {, } \\
& \begin{array}{lll}
\widetilde{E}_{n-2}=1-E_{n-2}, & \widetilde{E}_{n-1}=-2 \beta-E_{n-1}, & \widetilde{E}_{j}=-E_{j}, j=1, \ldots, n-3 \\
\widetilde{F}_{n-1}=-2 \beta-F_{n-1}, & \widetilde{F}_{n-2}=1-F_{n-2}, & \widetilde{F}_{j}=-F_{j}, j=1, \ldots, n-3 \\
\widetilde{f}_{n+1}=-x f_{n}, & \widetilde{f}_{n+1,1}=-2 \beta+x f_{n} w_{n-1}, & \widetilde{f}_{n+1,2}=1+x f_{n} w_{n-2} \\
\widetilde{p}_{2 n}=-x p_{2 n-1}, & \widetilde{p}_{2 n, 1}=-x p_{2 n-1}-F_{n}, & \widetilde{p}_{2 n, 2}=-x p_{2 n-1}+x f_{n} w_{n} .
\end{array}
\end{aligned}
$$

Denote this last $(2 n-2) \times(2 n-2)$ matrix as $M_{2 n-2}$. Note that $A, B, C, D, \delta,-c w_{1}$, $-2 \beta, \sigma_{i}$ are constants, $\widetilde{p}_{2 n, 1}, \widetilde{p}_{2 n, 2}$ and $\widetilde{p}_{2 n}$ are polynomials of degree $2 n$, all the other letters denote polynomials of degree $n+1$. 
Since by definition the determinant of a matrix $\left(a_{i j}\right)$ of order $m$ is

$$
\sum_{\sigma\left(j_{1}, \ldots, j_{m}\right)}(-1)^{\tau\left(j_{1}, \ldots, j_{m}\right)} a_{1 j_{1}} \ldots a_{m j_{m}},
$$

where $\sigma$ is a permutation of $\{1, \ldots, m\}$ and the summation is taken over all these possible permutations, and $\tau$ evaluated on a permutation of $\{1, \ldots, m\}$ is the minimum number of transpositions for passing the permutation to the identity, for calculating the degree of this determinant, we must first compute the highest degree terms $a_{1 j_{1}} \ldots a_{m j_{m}}$ in the summation. In order to realize this aim we must choose the $a_{i j_{i}}$ 's in such a way that the product has the highest possible degree. Applying this idea to the determinant of the matrix $M_{2 n-2}$, in the row and column where $\widetilde{p}_{2 n, 1}, \widetilde{p}_{2 n, 2}, \widetilde{p}_{2 n}$ is located, we must choose either one of these three polynomials or the two ones on the row and column where one of these three polynomials is located, because their product has the highest degree. The details are the following:

- For $\widetilde{p}_{2 n, 1}$ and $\widetilde{p}_{2 n, 2}$ we can choose respectively $G_{1} \widetilde{F}_{1}$ and $\widetilde{E}_{1} x f_{n} w_{1}$. But we can choose only one of them, because $\widetilde{F}_{1}$ and $x f_{n} w_{1}$ are located on the same column. So in the product we choose $G_{1} \widetilde{F}_{1}$ and $\widetilde{p}_{2 n, 2}$. Of course we can also choose $\widetilde{p}_{2 n, 1}$ and $\widetilde{E}_{1} x f_{n} w_{1}$ as the elements in the product.

- Next in order to get the highest degree in the product, we must choose the $B$ or $A$ in the second row, which is associated to $\widetilde{p}_{2 n, 1}$. We choose $A$.

- Then for the first $\widetilde{p}_{2 n}$ from above, we choose $\widetilde{f}_{n+1}$ and $\widetilde{E}_{1}$, which are located respectively on the row and column where the first $\widetilde{p}_{2 n}$ is located.

- For the second $\widetilde{p}_{2 n}$, we can only choose itself, because there are no other two elements on the row and column where the second $\widetilde{p}_{2 n}$ is located, whose product has degree larger than $2 n$.

- Correspondingly in the fourth row we can choose either the $B$ or the $A$, we choose $A$.

- To the third $\widetilde{p}_{2 n}$, we choose $\widetilde{f}_{n+1}$ and $\widetilde{E}_{1}$, which are located respectively on the row and column where the third $\widetilde{p}_{2 n}$ is located.

- In the row and column where the fourth $\widetilde{p}_{2 n}$ are located, we can choose only $\widetilde{p}_{2 n}$ itself as an element of the product.

- Then in the sixth row we can choose only either the $B$ or the $A$, and for the fifth $\widetilde{p}_{2 n}$ we must choose $\widetilde{f}_{n+1}$ and $\widetilde{E}_{1}$ as the elements of the product.

- According to this algorithm we get the highest degree terms in the summation in the following products

$-n$ polynomials of degree $n+1$ and $(n-2) / 2$ polynomials of degree $2 n$ provided that $n$ is even,

$-n-1$ polynomials of degree $n+1$ and $(n-1) / 2$ polynomials of degree $2 n$ provided that $n$ is odd.

Summarizing we have proved that the highest degree of $R_{L}$ is at most

$$
\begin{array}{ll}
n(n+1)+\frac{n-2}{2} 2 n=2 n^{2}-n & \text { if } n \text { is even, } \\
(n-1)(n+1)+\frac{n-1}{2} 2 n=2 n^{2}-n-1 & \text { if } n \text { is odd. }
\end{array}
$$


Note that this last two numbers are both even, and recall that they are denoted $2 d_{n}$.

The above proof provides the maximum degree of the polynomial $R_{L}$ for $n>3$. We now study the degree of $R_{L}$ for $n=2,3$, and for these values of $n$ we present the explicit expressions of $R_{L}$.

For $n=2$ we work in a similar way as we did for $n>3$, and we get that

$$
R_{L}=-(8 a b)^{3} \operatorname{det}\left(M_{4}^{*}-M_{3}^{*}\right),
$$

where

$$
\begin{aligned}
& M_{4}^{*}=\left[\begin{array}{cc}
p_{3}(x) & q_{2}(x) \\
-x p_{3}(x) & -2 \beta
\end{array}\right], \\
& M_{3}^{*}=\left[\begin{array}{cc}
-\eta_{1} f_{2}+\left(\eta_{2}-A^{-2} D\right) g_{1}+(2 \alpha+1) A^{-1} g_{1} & A^{-1} f_{2}-\eta_{1} g_{1} \\
\eta_{1} x f_{2}+\left(\eta_{2}-A^{-2} D\right) D+(2 \alpha+1) A^{-1} D & -A^{-1} x f_{2}-\eta_{1} D
\end{array}\right],
\end{aligned}
$$

with $\eta_{1}=A^{-2}(B+C)$ and $\eta_{2}=A^{-3}(B+C)^{2}$. Since $q_{2}(x)-\left(A^{-1} f_{2}(x)-\eta_{1} g_{1}(x)\right)=$ $A^{-2}(B+C)^{2}-A^{-1} D+2 \alpha+1$, it follows that $R_{L}$ is of degree 6 with the monomial $x^{6}$ of higher degree, which comes from the product of the monomials $x^{3}$ of higher degree in the polynomials $p_{3}(x)$ and $A^{-1} x f_{2}(x)$.

For $n=3$ similar computations yield

$$
R_{L}=(8 a b)^{4} \operatorname{det}\left(M_{4}^{*}-M_{3}^{*}\right)
$$

with

$$
\begin{aligned}
M_{4}^{*}= & {\left[\begin{array}{cccc}
A & q_{4}(x) & r_{3}(x) & x^{2} \\
B & -2 \beta & 1 & 2 \alpha \\
C & -x p_{5}(x) & -2 \beta & 1 \\
D & 0 & -x p_{5}(x) & -2 \beta
\end{array}\right], } \\
M_{3}^{*}= & {\left[\begin{array}{cccc}
0 & -A^{-2} B f_{3}+u_{3} g_{2}+\gamma_{4} h_{1} & A^{-1} f_{3}-A^{-2} B g_{2}+u_{3} h_{1} & A^{-1} g_{2}-A^{-2} B h_{1} \\
0 & A^{-2} B x f_{3}+u_{3} D+\gamma_{4} C & -A^{-1} x f_{3}-A^{-2} B D+u_{3} C & A^{-1} D-A^{-2} B C \\
0 & -u_{3} x f_{3}+\gamma_{4} D & A^{-2} B x f_{3}+u_{3} D & -A^{-1} x f_{3}-A^{-2} B D \\
0 & -\gamma_{4} x f_{3} & -u_{3} x f_{3} & A^{-2} B x f_{3}
\end{array}\right], }
\end{aligned}
$$

where $\gamma_{4}=-A^{-4} B^{3}+2 A^{-3} B C-A^{-2} D+2 \alpha A^{-1}$. Note that $A^{-1} g_{2}-A^{-2} B h_{1}=$ $x^{2}-A^{-2} B^{2}+A^{-1} C$. Some additional calculations show that polynomial entries $m_{i 1}^{*}$ for $i=1, \ldots, 4, m_{13}^{*}$ and $m_{14}^{*}$ of the matrix $M_{4}^{*}-M_{3}^{*}:=\left(m_{i j}^{*}(x)\right)_{4 \times 4}$ have degree zero, and that the degree of the polynomials $m_{32}^{*}$ and $m_{43}^{*}$ is 6 . All the other entries have degree 4 . In the determinant of the matrix $M_{4}^{*}-M_{3}^{*}$, the product containing the two entries of degree 6 provides only degree 12 . Taking one degree 6 entry and two entries of degree 4 we get the maximum degree 14 .

This completes the proof of the lemma.

Proof of statement (a) Theorem 2. From (3) we have that for each solution $\left(x_{L}, x_{R}\right)$ with $x_{L} \neq x_{R}$ of the polynomial systems $E_{L}=0$ and $E_{R}=0$, we have a crossing limit cycle of the discontinuous piecewise linear differential system (2) which intersect the curve $y=x^{n}$ at the points $\left(x_{L}, x_{L}^{n}\right)$ and $\left(x_{R}, x_{R}^{n}\right)$.

Recall that we denote by $2 d_{n}$ the degree of the resultant $R_{L}$ computed in Lemma 3. Of course, the expression of $2 d_{n}$ changes according the parity of $n$. 
If $\left(x_{L}, x_{R}\right)$ with $x_{L} \neq x_{R}$ is a solution of the polynomial system $E_{L}=0$ and $E_{R}=0$, the value $x_{L}$ is a root of the resultant $R_{L}$, and the value $x_{R}$ is a root of the resultant $R_{R}$. But both resultants $R_{L}$ and $R_{R}$ have the same roots, because these two polynomials are the same, we can pass from one to the other interchanging the variable $x_{L}$ by the variable $x_{R}$. So the possible values for $x_{L}$ and for $x_{R}$ are the same. Consequently we only have at most $2 d_{n}$ points $\left(x_{L}, x_{L}^{n}\right)$ and $\left(x_{R}, x_{R}^{n}\right)$, all together, which can be points where the crossing limit cycles intersect the curve $y=x^{n}$. Hence we cannot have more then $d_{n}$ crossing limit cycles. This completes the proof of statement $(a)$ of the theorem.

Proof of statement (b) Theorem 2. We provide an example showing that the upper bound of 3 limit cycles given in Theorem 2 for the maximum number of limit cycles that a discontinuous piecewise linear differential system in $\mathbb{R}^{2}$ separated by the parabola $y=x^{2}$ when both linear differential systems have a center is reached. Consider the linear differential system

$$
\begin{aligned}
& \dot{x}=-\frac{189 x}{100}+\frac{38221 y}{20500}-\frac{720042289}{205000000}, \\
& \dot{y}=-\frac{41 x}{20}+\frac{189 y}{100}-\frac{3296401}{1000000},
\end{aligned}
$$

in the region $\mathrm{L}$, and the linear differential system

$$
\begin{aligned}
& \dot{x}=\frac{18}{5}-y, \\
& \dot{y}=x-\frac{99}{50},
\end{aligned}
$$

in the region U. Both linear differential systems form a discontinuous piecewise linear differential system in $\mathbb{R}^{2}$ separated by the parabola $y=x^{2}$ having two centers. This discontinuous piecewise linear differential system has the three limit cycles of the Figure 1.

We note that the value of the coordinate $x$ of the six points $\left(x, x^{2}\right)$ of the parabola which belong to the limit cycles are the six real roots of the polynomial $R_{L}$ equal to

$$
\begin{aligned}
& 10769750976621134140624876981-29736678501017804062959688750 x+ \\
& 2515419213211056546308719750 x^{2}+25945028877701413073475000000 x^{3}- \\
& 5566633022238812671927500000 x^{4}-5065968152418838875000000000 x^{5}+ \\
& 1454075658590625000000000000 x^{6} .
\end{aligned}
$$

The discontinuous piecewise linear differential system (4)-(5) proves the statement (b) of Theorem 2.

Proof of statement (c) Theorem 2. For $n=2$ we have that $d_{n}=3$, and consequently by Theorem 2 the discontinuous piecewise linear differential system (2) will have at most 3 limit cycles. The concrete expression of the resultant $R_{L}$ modulo the coefficient of the highest degree monomial is

$$
R_{L}^{*}=x^{6}+C_{1} x^{5}+C_{2} x^{4}+C_{3} x^{3}+C_{4} x^{2}+C_{5} x+C_{6},
$$


as before we have replaced the variable $x_{L}$ by the variable $x$ for simplicity, and

$$
\begin{aligned}
C_{1}= & 2 A \eta_{1}, \\
C_{2}= & A^{-1} D+2 A^{2} \eta_{1}^{2}+\eta_{3}, \\
C_{3}= & 4 D \eta_{1}-4 \beta \\
C_{4}= & A^{-2} D^{2}-A^{-1} D \eta_{3}+D \eta_{2}+\eta_{3}^{2}+A \eta_{1}\left(D \eta_{1}-4 \beta\right)+A^{2} \eta_{1}^{2} \eta_{3}, \\
C_{5}= & 2 A^{-1} D\left(D \eta_{1}-\beta\right)-2 \beta \eta_{3}-2 A D \eta_{1} \eta_{2}-2 A^{2}\left(\beta-D \eta_{1}\right) \eta_{1}^{2}, \\
C_{6}= & A^{-3} D^{3}-2 A^{-2} D^{2} \eta_{3}+A^{-1} D\left(\eta_{3}+1\right) \eta_{3}-D \eta_{1}\left(6 \beta+D \eta_{1}-\eta_{1} \eta_{3}\right) \\
& +4 \beta^{2}-A\left(D \eta_{2}^{2}-2 \beta \eta_{1} \eta_{3}\right)+A^{3} \eta_{1}^{3}\left(2 \beta+D \eta_{1}\right),
\end{aligned}
$$

where $\eta_{3}=2 \alpha+1$.

For $n=3$ we have that $d_{n}=7$, therefore by Theorem 2 the discontinuous piecewise linear differential system (2) will have at most 7 limit cycles. The resultant $R_{L}=$ Resultant $\left[E_{L}\left(x_{L}, x_{R}\right), E_{R}\left(x_{L}, x_{R}\right), x_{R}\right]$ has a huge expression directly using the parameters of the system. Here we choose the previous notations $A, B, C, D$, and still replace $x_{L}$ by $x$ for simplicity, we get the next formula $R_{L}^{*}$ of $R_{L}$ modulo a multiplicative constant.

$$
\begin{aligned}
R_{L}^{*}= & C_{0} x^{14}+C_{1} x^{13}+C_{2} x^{12}+C_{3} x^{11}+C_{4} x^{10}+C_{5} x^{9}+C_{6} x^{8}+C_{7} x^{7}+C_{8} x^{6} \\
& +C_{9} x^{5}+C_{10} x^{4}+C_{11} x^{3}+C_{12} x^{2}+C_{13} x+C_{14},
\end{aligned}
$$

where

$$
\begin{aligned}
C_{0}= & -3 B \\
C_{1}= & 6 A^{-1} B^{2}, \\
C_{2}= & -4 A^{-2} B^{3}-2 A^{-1} B(C+2 D)-3 D+4 A \alpha \\
C_{3}= & -2 A^{-2} B^{2}(C+5 D)-12 A^{-1} B D \\
C_{4}= & -2 A^{-3} B^{3}(3 C-5 D)-18 A^{-2} B^{2} D-A^{-2} B D(8 C+D) \\
& -2 A^{-1}\left(3 B^{2} \alpha+2 C D+D^{2}\right)-7 B+6 C \alpha+4 D \alpha-4 A \beta, \\
C_{5}= & 4 A^{-4} B^{4}(C-D)-2 A^{-3} B^{2}\left(4 B D-5 C^{2}+10 C D+D^{2}\right) \\
& +2 A^{-2} B\left(4 B^{2} \alpha-5 C D+7 D^{2}\right)-2 A^{-1}\left(5 B^{2}+6 B C \alpha-3 D^{2}\right) \\
& +2 B \beta+2 D \alpha+8 A \alpha^{2}, \\
C_{6}= & A^{-4} B^{3}\left(15 C^{2}-18 C D-D^{2}\right)+2 A^{-3} B\left(B C D-13 B D^{2}+2 C^{3}-4 C^{2} D-C D^{2}\right) \\
& -A^{-2}\left(4 B^{3}-4 B^{2} C \alpha-8 B^{2} D \alpha-18 B D^{2}-6 C D^{2}-3 C^{2} D\right) \\
& +2 A^{-1}\left(3 B^{2} \beta-B C-3 B D+3 C^{2} \alpha+4 C D \alpha+D^{2} \alpha\right)-7 D+6 C \beta+4 D \beta+8 A \alpha, \\
C_{7}= & A^{-5} B^{4} C(C-D)+2 A^{-4} B^{2}\left(8 B C D^{2}+8 C^{3}-10 C^{2} D-C D^{2}-10 D^{2}\right) \\
& +2 A^{-3} B\left(B^{3}-4 B^{2} D \alpha-9 B D^{2}+C^{2} D-12 C D^{2}-D^{3}\right) \\
& -2 A^{-2}\left(B^{3} \beta+5 B^{2} D+9 B^{2} D \alpha-2 B C^{2} \alpha-2 B C D \alpha-2 B D^{2} \alpha+4 C D^{2}+2 D^{3}\right) \\
& \left.-4 A^{-1}\left(B C \beta+B D \beta+5 B D-3 C D \alpha-D^{2} \alpha\right)+8 D \alpha^{2}\right)-16 A \alpha \beta, \\
C_{8}= & A^{-5} B^{3}\left(2 B D+5 C^{2}\right)(C-D)+4 A^{-4} B^{4}(C-D) \alpha-4 A^{-4} B^{3} D^{2} \\
& +2 A^{-4} B^{2}\left(15 C^{2} D-20 C D^{2}-D^{3}\right)+A^{-4} B C^{2}\left(4 C^{2}-D^{2}-6 C D\right) \\
& -8 A^{-3} B^{4} \beta+2 A^{-3} B^{3}(5 C-2 D-8 D \alpha)-2 A^{-3} B^{2}\left(5 C^{2}+2 C D-D^{2}\right) \alpha \\
& -2 A^{-3} B D^{2}(8 C+7 D)-2 A^{-3} C D\left(C^{2}+C D+D^{2}\right)-4 A^{-2} B^{3} \alpha^{2} \\
& +2 A^{-2} B^{2}(3 C \beta-11 D-2 D \beta)-A^{-2} B\left(8 C D-26 C D \alpha+D^{2}+8 D^{2} \alpha\right) \\
& +2 A^{-2}\left(2 C^{3} \alpha+2 C^{2} D \alpha+3 C D^{2} \alpha-2 D^{3}\right)-2 A^{-1} B^{2} \alpha \\
& +2 A^{-1} B\left(2 C \alpha^{2}+4 D \alpha^{2}+3 D \beta\right)-2 A^{-1}\left(2 C^{2} \beta+3 C D+6 C D \beta+D^{2}+D^{2} \alpha\right) \\
& -5 B-4 B \alpha \beta+8 C \alpha+4 D \alpha+4 D \alpha^{2}+8 A\left(\alpha^{3}-\beta\right),
\end{aligned}
$$




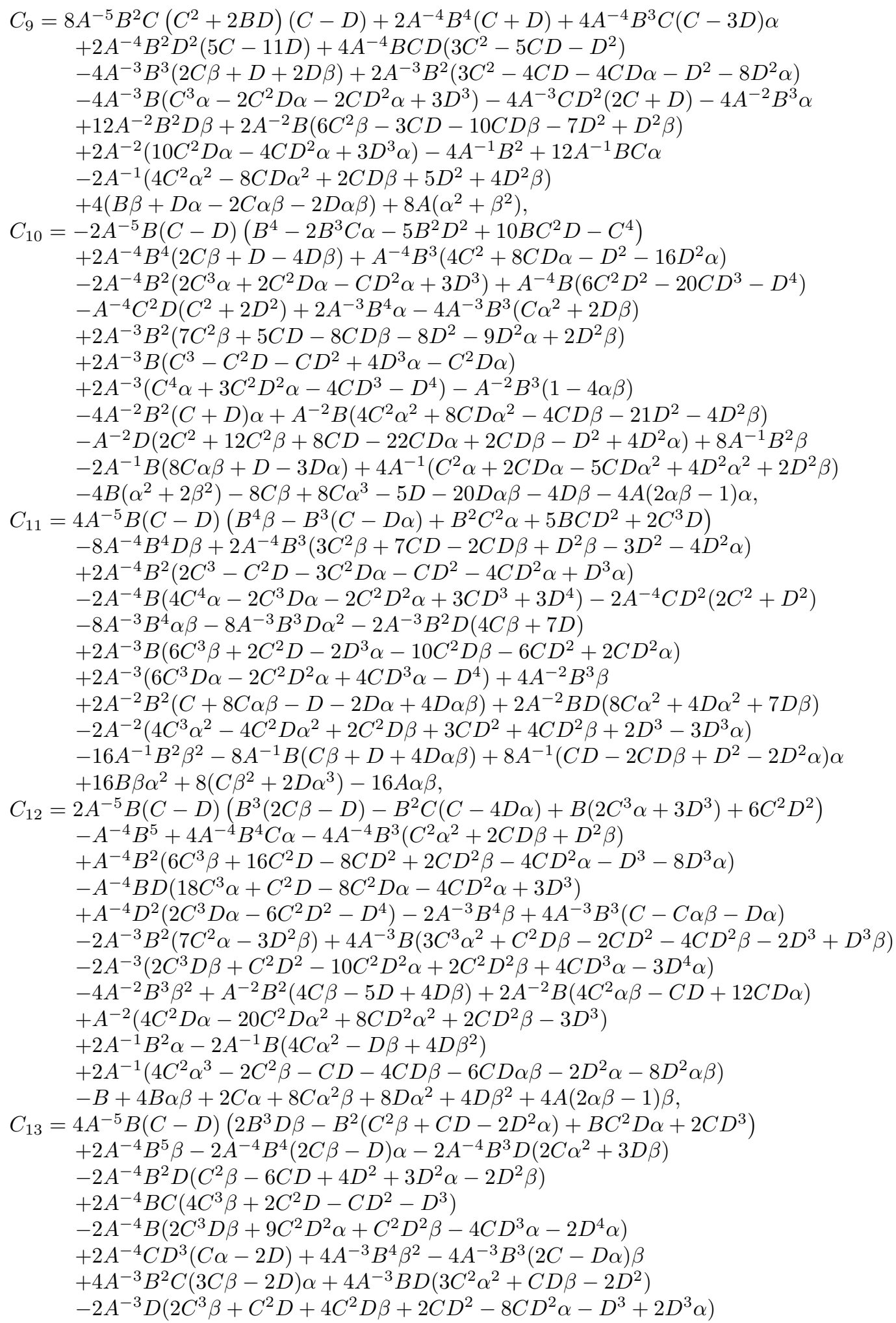




$$
\begin{aligned}
& -2 A^{-2} B^{2}\left(4 C \beta^{2}-5 D \beta+4 D \beta^{2}\right)+2 A^{-2} B\left(2 C^{2} \beta+C D-14 C D \beta \alpha-D^{2}+3 D^{2} \alpha\right) \\
& +4 A^{-2}\left(2 C^{3} \alpha \beta+C^{2} D \alpha-2 C^{2} D \alpha \beta-5 C D^{2} \alpha^{2}+2 D^{3} \alpha^{2}+D^{3} \beta\right) \\
& -4 A^{-1} B^{2} \alpha \beta+4 A^{-1} B\left(2 C \alpha^{2} \beta-D \alpha^{2}-D \beta^{2}\right) \\
& +4 A^{-1}\left(2 C^{2} \beta^{2}-C D \beta+2 C D \alpha^{3}-D^{2}-2 D^{2} \beta-D^{2} \alpha \beta\right) \\
& \left.+2\left(B \beta-4 B \alpha \beta^{2}+D \alpha-4 D \alpha^{2} \beta\right)+8 A \beta^{2}\right), \\
C_{14}= & 2 A^{-5} B(C-D)\left(B^{2} D(4 C \beta+D)-B C\left(2 C^{2} \beta+C D-2 D^{2} \alpha\right)+D^{4}\right) \\
& -4 A^{-4} B^{5} \beta^{2}-A^{-4} B^{4}(2 C \beta+D+8 D \alpha \beta)+2 A^{-4} B^{3}\left(2 C^{2} \beta+C D-2 D^{2} \alpha\right) \alpha \\
& -2 A^{-4} B^{2} D^{2}(9 C \beta+D-4 D \beta) \\
& +A^{-4} B D\left(10 C^{3} \beta+5 C^{2} D-4 C^{2} D \beta-10 C D^{2} \alpha-2 C D^{2}+4 C D^{2} \beta+D^{4}+4 D^{3} \alpha\right) \\
& -A^{-4} D^{2}\left(2 C^{3} \beta+C^{2} D-2 C D^{2} \alpha+D^{3}\right) \\
& +2 A^{-3} B^{3}\left(10 C \beta^{2}+D \beta\right)+2 A^{-3} B^{2}\left(4 C^{2} \beta+2 C D+12 C D \alpha \beta-D^{2} \alpha\right) \\
& -2 A^{-3} B\left(6 C^{3} \alpha \beta+3 C^{2} D \alpha-6 C D^{2} \alpha^{2}-5 D^{3} \beta\right) \\
& -2 A^{-3} D^{2}\left(2 C^{2} \beta+C D+2 C D \beta+D^{2}-3 D^{2} \alpha+D^{2} \beta\right) \\
& -20 A^{-2} B^{2} D \beta^{2}-2 A^{-2} B\left(6 C^{2} \beta^{2}+7 C D \beta+4 C D \beta^{2}+2 D^{2}+14 D^{2} \alpha \beta\right) \\
& +2 A^{-2} D\left(6 C^{2} \alpha \beta-2 C^{2} \beta-C D+3 C D \alpha+2 D^{2} \alpha-6 D^{2} \alpha^{2}\right) \\
& +8 A^{-1} B^{2} \alpha \beta^{2}+2 A^{-1} B(2 C \beta+D+8 D \alpha \beta) \alpha \\
& -2 A^{-1}\left(4 C^{2} \alpha^{2} \beta+2 C D \alpha^{2}-6 C D \beta^{2}-4 D^{2} \alpha^{3}-3 D^{2} \beta-4 D^{2} \beta^{2}\right) \\
& -4 B \beta^{2}-2 C \beta-16 C \alpha \beta^{2}-D-12 D \alpha \beta-8 A \beta^{3},
\end{aligned}
$$

where $u_{3}=A^{-3} B^{2}-A^{-2} C$ and $\eta_{4}=-A^{-4} B^{3}+2 A^{-3} B C-A^{-2} D+2 A^{-1} \alpha$. This completes the proof of statement (c) of Theorem 2 .

\section{ACKNOWLEDGEMENTS}

The first author is partially supported by the MINECO grants MTM2016-77278$\mathrm{P}$ and MTM2013-40998-P, an AGAUR grant 2014SGR-568, and the grant FP7PEOPLE-2012-IRSES 318999, and from the recruitment program of high-end foreign experts of China.

The second author is partially supported by NNSF of China grants 11271252 and 11671254, and by innovation program of Shanghai municipal education commission grant $15 Z Z 012$.

\section{REFERENCES}

[1] A. Andronov, A. Vitt and S. Khaikin, Theory of Oscillations, Pergamon Press, Oxford, 1966.

[2] J.C. Artés, J. Llibre, J.C. Medrado and M.A. Teixeira, Piecewise linear differential systems with two real saddles, Math. Comp. Sim. 95 (2013), 13-22.

[3] M. di Bernardo, C.J. Budd, A.R. Champneys, P. Kowalczyk, Piecewise-Smooth Dynamical Systems: Theory and Applications, Appl. Math. Sci. Series 163, Springer-Verlag, London, 2008.

[4] D.C. BRaga And L.F. Mello, Limit cycles in a family of discontinuous piecewise linear differential systems with two zones in the plane, Nonlinear Dynam. 73 (2013), 1283-1288.

[5] D.C. Braga And L.F. Mello, More than three limit cycles in discontinuous piecewise linear differential systems with two pieces in the plane, Internat. J. Bifur. Chaos 24 (2014), 1450056, pp. 10.

[6] C. Buzzi, C. Pessoa And J. Torregrosa, Piecewise linear perturbations of a linear center, Discrete Contin. Dyn. Syst. 9 (2013), 3915-3936.

[7] R.D. EuzÉBIO AND J. LliBRE, On the number of limit cycles in discontinuous piecewise linear differential systems with two zones separated by a straight line, J. Math. Anal. Appl. 424 (2015), 475-486. 
[8] A.F. Filippov, Differential equations with discontinuous right-hand sides, Nauka, Moscow, 1985 (transl. Kluwer, Dordrecht, 1988).

[9] E. Freire, E. Ponce, F. Rodrigo And F. Torres, Bifurcation sets of continuous piecewise linear systems with two zones, Internat. J. Bifur. Chaos 8 (1998), 2073-2097.

[10] E. Freire, E. Ponce And F. Torres, Canonical discontinuous planar piecewise linear systems, SIAM J. Appl. Dynam. Syst. 11 (2012), 181-211.

[11] E. Freire, E. Ponce And F. Torres, A general mechanism to generate three limit cycles in planar Filippov systems with two zones, Nonlinear Dynam. 78 (2014), 251-263.

[12] F. Giannakopoulos And K. Pliete, Planar systems of piecewise linear differential equations with a line of discontinuity, Nonlinearity 14 (2001), 1611-1632.

13] M. HAN AND W. Zhang, On Hopf bifurcation in non-smooth planar systems J. Differential Equations 248 (2010), 2399-2416.

[14] D. Hilbert, Mathematische Probleme, Lecture, Second Internat. Congr. Math. (Paris, 1900), Nachr. Ges. Wiss. Göttingen Math. Phys. KL. (1900), 253-297; English transl., Bull. Amer. Math. Soc. 8 (1902), 437-479; Bull. (New Series) Amer. Math. Soc. 37 (2000), 407-436.

[15] S.M. HuAN AND X.S. YANG, On the number of limit cycles in general planar piecewise linear systems, Discrete Contin. Dynam. Syst. Ser. A 32 (2012), 2147-2164.

[16] S.M. HUAN AND X.S. YANG, On the number of limit cycles in general planar piecewise linear systems of node-node types, J. Math. Anal. Appl. 411 (2013), 340-353.

[17] S.M. HuAN AND X.S. YANG, Existence of limit cycles in general planar piecewise linear systems of saddle-saddle dynamics, Nonlinear Anal. 92 (2013), 82-95.

[18] Yu. Ilyashenko, Centennial history of Hilbert's 16th problem, Bull. Amer. Math. Soc. 39 (2002), 301-354.

[19] E.M. Izhikevich, Dynamical Systems in Neuroscience: the Geometry of Excitability and Bursting, Comput. Neurosci., MIT Press, Cambridge, MA, 2007.

[20] P. Kokotović, H.K. Khalil and J. O'Reilly, Singular Perturbation Methods in Control: Analysis and Design, Classics Appl. Math., SIAM, Philadelphia, PA, 1999.

[21] A. LÉGER AND E. PRATT, Qualitative analysis of a forced nonsmooth oscillator with contact and friction, Ann. Solid Struct. Mech. 2 (2011), 1-17.

[22] J. LI, Hilbert's 16th problem and bifurcations of planar polynomial vector fields, Internat. J. Bifur. Chaos 13 (2003), 47-106.

[23] L. LI, Three crossing limit cycles in planar piecewise linear systems with saddle-focus type, Electron. J. Qual. Theory Differ. Equ. 2014, no. 70, pp. 14.

[24] J. Llibre, D.D. Novaes AND M.A. TeixeIRA, Maximum number of limit cycles for certain piecewise linear dynamical systems, Nonlinear Dynam. 82 (2015), 1159-1175.

[25] J. Llibre, D.D. Novaes, M.A. TeIXeIRA, Limit cycles bifurcating from the periodic orbits of a discontinuous piecewise linear differential center with two zones, Internat. J. Bifur. Chaos 25 (2015), 1550144, pp. 11.

[26] J. Llibre And E. POnCE, Three nested limit cycles in discontinuous piecewise linear differential systems with two zones, Dynam. Contin. Discrete Impul. Syst. Ser. B 19 (2012), $325-335$.

[27] J. Llibre, M.A. Teixeira And J. Torregrosa, Lower bounds for the maximum number of limit cycles of discontinuous piecewise linear differential systems with a straight line of separation, Internat. J. Bifur. Chaos 23 (2013), 1350066, pp. 10.

[28] J. LliBRE AND X. ZHANG, Limit cycles for discontinuous planar piecewise linear differential systems, preprint, 2016

[29] O. Makarenkov and J.S.W. Lamb, Dynamics and bifurcations of nonsmooth systems: A survey, Physica D 241 (2012), 1826-1844.

[30] D.D. Novaes And E. Ponce, A simple solution to the Braga-Mello conjecture, Internat. J. Bifur. Chaos 25 (2015), 1550009, pp. 7.

[31] E. Pratt, A. LÉGer AND M. JEAN, About a stability conjecture concerning unilateral contact with friction, Nonlinear Dynam. 59 (2010), 73-94.

[32] S. Shui, X. Zhang AND J. LI, The qualitative analysis of a class of planar Filippov systems, Nonlinear Anal. 73 (2010), 1277-1288.

[33] D.J.W. Simpson, Bifurcations in Piecewise-Smooth Continuous Systems, World Scientific Series on Nonlinear Science A, vol 69, World Scientific, Singapore, 2010.

[34] M.A. TeIXeIrA, Perturbation theory for non-smooth systems, In Mathematics of complexity and dynamical systems. Vols. 1-3, 1325-1336 pp., Springer, New York, 2012. 
[35] M. Wechselberger And W. Weckesser, Bifurcations of mixed-mode oscillations in a stellate cell model, Physica D 238 (2009), 1598-1614.

[36] M.A. TeIXeIra, Perturbation theory for non-smooth systems, in Encyclopedia of complexity and systems science, R. A. Meyers and G. Gaeta, Eds. Springer-Verlag, New York, 2009, 6697-6709.

1 Departament de Matemàtiques, Universitat Autònoma de Barcelona, 08193 Bellaterra, Barcelona, Catalonia, Spain

E-mail address: jllibre@mat.uab.cat

2 Department of Mathematics, MOE-LSC, Shanghai Jiao Tong University, Shanghai, 200240, P. R. China

E-mail address: xzhang@sjtu.edu.cn 